\title{
Pinning adaptive synchronization of neutral-type coupled neural networks with stochastic perturbation
}

\author{
Xiaojie Sun ${ }^{1 *}$, Zuhong Feng ${ }^{2}$ and Xueliang Liu ${ }^{3}$
}

\section{"Correspondence:}

sunxiaojie_2009@126.com

'Science and Technology

Information, Shaanxi Railway

Institute, Weinan, 714000, P.R. China

Full list of author information is

available at the end of the article

\section{Springer}

\begin{abstract}
In this paper, by using a pinning adaptive control scheme, we investigate the almost surely synchronization of neutral-type coupled neural networks with stochastic perturbation. Based on Lyapunov stability theory, stochastic analysis, and matrix theory, some sufficient conditions for almost surely synchronization are derived. Furthermore, a numerical example is exhibited to illustrate the validity of the theoretical results.
\end{abstract}

Keywords: synchronization; neutral-type; neural networks; adaptive pinning control; stochastic perturbation

\section{Introduction}

In recent years, the neural networks (NNs) have played a significant role in the fields of science and engineering due to their practical applications including signal and image processing, associative memories, combinatorial optimization, automatic control, and so on (see [1-4]). Among dynamical behaviors of the neural networks, synchronization is one of the most important topics that have received considerable research attention [5-9]. At the same time, many different kinds of synchronization have been proposed, such as generalized synchronization [9], cluster synchronization [10], phase synchronization [11], lag synchronization [12], and so on.

Due to the finite speed of transmitting signals between neurons and the transmission process randomly perturbed by the environmental elements, time delays and stochastic noises exist in various neural networks, which have become one of the main sources for causing instability and poor performance of neural networks. So far, most of the existing results related to the synchronization analysis for neural networks have concerned time delays and stochastic noises; see $[2,5-7,12-14]$ and the references therein.

In the case where the network cannot synchronize by itself, many control techniques have been developed to drive the network to achieve synchronize, such as linear state feedback control [15, 16], state observer-based control [17], impulsive control [18], and adaptive control [12]. All of them have the feature that the controller needs to be added to each node. But in practice, it is too difficult to add controllers to all nodes in a largescale network. To reduce the number of controlled nodes, pinning control is introduced, in which controllers are only applied to partial nodes. In [19], it has been shown that a single controller can ensure that the whole network synchronizes asymptotically with large

2014 Sun et al: licensee Springer. This is an Open Access article distributed under the terms of the Creative Commons Attribution License (http://creativecommons.org/licenses/by/2.0), which permits unrestricted use, distribution, and reproduction in any medium, provided the original work is properly cited. 
enough coupling strength and without any prior knowledge of the structure of the network topology. In [10], the cluster synchronization issue is considered for a class of delayed coupled complex dynamical networks by using the pinning control strategy. In addition, the pinning adaptive control method has received considerable research attention, which is utilized to get the appropriate control gains effectively. By using the adaptive pinning approach, the robust synchronization of a class of nonlinearly coupled complex networks is investigated in [20]. An adaptive pinning control method is proposed in [21] to synchronize a delayed complex dynamical network with free coupling matrix. In [22], the adaptive pinning synchronization is investigated for complex networks with non-delayed and delayed couplings and vector-form stochastic perturbations.

Recently, the stability and synchronization of neutral-type systems have been studied widely [23-27], in which the time delays occur not only in the system states but also in the derivatives of the system states. In [25], the problem of stochastic stability of neutraltype neural networks with Markovian jumping parameters is considered. By using the adaptive control approach, the exponential synchronization in the $p$ th moment of neutraltype delayed neural networks is investigated in [26]. In [27], an adaptive control method is proposed to synchronize for a class of coupled neutral-type complex dynamical networks by adding adaptive controller to all nodes. To the best of our knowledge, the problem of adaptive pinning synchronization for neutral-type neural networks with stochastic perturbation has received very little research attention.

In this paper, we are concerned with the analysis issue for adaptive pinning synchronization of neutral-type neural networks with stochastic perturbation. By using Lyapunov stability theory and adaptive pinning control approach, several criteria are given to guarantee the almost surely synchronization of neutral-type coupled neural networks with stochastic perturbation. A numerical example is also presented to show the effectiveness of the proposed method. The main contributions of this paper are as follows:

(1) A new class of neutral-type neural networks with pinning adaptive controller is considered.

(2) A new pinning adaptive law is designed.

The notations are quite standard. Throughout this paper, $R_{+}, R^{n}$, and $R^{n \times m}$ denote the set of non-negative real numbers, $n$ dimensional Euclidean space and the set of all $n \times m$ real matrices, respectively. The superscript $T$ denotes matrix transposition, trace $(\cdot)$ denotes the trace of the corresponding matrix and $I$ denotes the identity matrix. $\|\cdot\|$ stands for the Euclidean norm in $R^{n}$. $\operatorname{diag}\{\cdots\}$ stands for the block diagonal matrix. Let $(\Omega, \mathcal{F}, \mathcal{P})$ be a complete probability space with a filtration $\left\{\mathcal{F}_{t}\right\}_{t \geq 0}$ satisfying the usual conditions (i.e. the filtration contains all $P$-null sets and is increasing and right continuous). Denote by $C_{\mathcal{F}_{0}}^{b}\left([-\tau, 0] ; R^{n}\right)$ the family of all $\mathcal{F}_{0}$-measurable, bounded, and $C^{b}\left([-\tau, 0] ; R^{n}\right)$-value random variables.

\section{Model and preliminaries}

Consider a complex system consisting of $N$ linearly coupled identical neutral-type neural networks. The $i$ th neutral-type neural network [27] can be described by the following differential equation:

$$
\dot{x}_{i}(t)-D \dot{x}_{i}(t-\tau(t))=-C x_{i}(t)+A f\left(x_{i}(t)\right)+B g\left(x_{i}(t-\tau(t))\right)+J(t),
$$


where $x_{i}(t)=\left[x_{i 1}(t), x_{i 2}(t), \ldots, x_{i n}(t)\right]^{T} \in R^{n}$ is the state vector associated with $n$ neurons, $f(\cdot)$ and $g(\cdot)$ denotes the neuron activation functions, $\tau(t)$ represents the time-varying delay with $0<\tau(t)<\tau, \dot{\tau}(t) \leq \bar{\tau}<1, A=\left(a_{i j}\right)_{n \times n}$ and $B=\left(b_{i j}\right)_{n \times n}$ are the connection weight and the delay connection weight matrices, respectively, $C=\operatorname{diag}\left\{c_{1}, c_{2}, \ldots, c_{n}\right\}$ is a positive diagonal matrix, $D=\left(d_{i j}\right)_{n \times n}$ is called the neutral-type parameter matrix, and $J(t) \in R^{n}$ is the constant external input vector.

The dynamical behavior of linearly coupled neutral-type neural networks with stochastic perturbation can be described by the following stochastic delayed differential equations:

$$
\begin{aligned}
d\left[x_{i}(t)-D x_{i}(t-\tau(t))\right] & \\
= & \left\{-C x_{i}(t)+A f\left(x_{i}(t)\right)+B g\left(x_{i}(t-\tau(t))\right)+J(t)+c \sum_{j=1}^{N} g_{i j} \Gamma x_{j}(t)+u_{i}(t)\right\} d t \\
& +\sigma\left(t, e_{i}(t), e_{i}(t-\tau(t))\right) d B(t), \quad i=1,2, \ldots, N,
\end{aligned}
$$

where $\Gamma=\operatorname{diag}\left\{\gamma_{1}, \gamma_{2}, \ldots, \gamma_{n}\right\}$ is a diagonal matrix describing the inner coupling between two connected nodes $i$ and $j(i \neq j)$ at time $t$; $c$ is the coupling strength; $G=\left(g_{i j}\right)_{N \times N}$ is the out coupling configuration matrix which represents the topological structure of the whole network, where the entries $g_{i j}$ are defined as follows: if there is a link from node $i$ to node $j$, then $g_{i j}=g_{j i}>0(j \neq i)$; Otherwise, $g_{i j}=g_{j i}=0$. It assumes that $\sum_{j=1}^{N} g_{i j}=0 ; u_{i}(t)$ is a control input vector, $B(t) \in R^{m}$ is an $m$-dimensional Brownian motion defined on a complete probability space $(\Omega, \mathcal{F}, \mathcal{P}), \sigma(\cdot, \cdot, \cdot): R_{+} \times R^{n} \times R^{n} \rightarrow R^{n \times m}$ is a Borel measurable matrix function, $e_{i}(t)=x_{i}(t)-s(t)$ denotes the error between the state of node $i$ and the desired state vector $s(t)$. The dynamical equations of $s(t)$ and $e_{i}(t)$ satisfy

$$
\begin{aligned}
\dot{s}(t)- & D \dot{s}(t-\tau(t))=-C s(t)+A f(s(t))+B g(s(t-\tau(t)))+J(t), \\
d\left[e_{i}(t)-D e_{i}(t-\tau(t))\right] & \\
= & \left\{-C e_{i}(t)+A f\left(e_{i}(t)\right)+B g\left(e_{i}(t-\tau(t))\right)+c \sum_{j=1}^{N} g_{i j} \Gamma e_{j}(t)+u_{i}(t)\right\} d t \\
& +\sigma\left(t, e_{i}(t), e_{i}(t-\tau(t))\right) d B(t), \quad i=1,2, \ldots, N,
\end{aligned}
$$

where $f\left(e_{i}(t)\right)=f\left(x_{i}(t)\right)-f(s(t))$ and $g\left(e_{i}(t-\tau(t))\right)=g\left(x_{i}(t-\tau(t))\right)-g(s(t-\tau(t)))$.

To prove our main results, the following assumptions are necessary.

Assumption 1 The functions $f(\cdot)$ and $g(\cdot)$ satisfy the Lipschitz condition. That is, there exist two positive constants $l_{1}$ and $l_{2}$, such that

$$
\begin{aligned}
& \|f(x)-f(y)\| \leq l_{1}\|x-y\|, \\
& \|g(x)-g(y)\| \leq l_{2}\|x-y\|
\end{aligned}
$$

hold, for any $x, y \in R^{n}$. 
Assumption 2 The noise intensity matrix $\sigma(\cdot, \cdot, \cdot)$ satisfies the bound condition. That is, there exist two positive constant $h_{1}$ and $h_{2}$, such that

$$
\operatorname{trace}\left[\sigma^{T}(t, x, y) \sigma(t, x, y)\right] \leq h_{1}\|x\|^{2}+h_{2}\|y\|^{2}
$$

hold, for any $x, y \in R^{n}$.

Assumption 3 There is a constant $\kappa \in(0,1)$ such that

$$
\|D x-D \bar{x}\| \leq \kappa\|x-\bar{x}\| \quad \forall x, \bar{x} \in R^{n} .
$$

In order to derive the main results, the following definitions and lemmas are necessary in this paper.

Consider a stochastic differential delays equation with the form

$$
d x(t)=f(t, x(t), x(t-\tau)) d t+g(t, x(t), x(t-\tau)) d \omega(t)
$$

on $t \in[0,+\infty)$ with the initial data given by $\xi \in C_{\mathcal{F}_{0}}^{b}\left([-\tau, 0] ; R^{n}\right)$.

Definition 1 [28] The trivial solution $x(t, \xi)$ of the system (5) is said to be almost surely asymptotically stable if

$$
P\left(\lim _{t \rightarrow \infty} x(t, \xi)=0\right)=1
$$

for all initial conditions $\xi \in C_{\mathcal{F}_{0}}^{b}\left([-\tau, 0] ; R^{n}\right)$.

Definition 2 The network (2) is said to have almost surely asymptotical synchronization if network (4) is almost surely asymptotically stable.

Lemma 1 [23] Assume that system (5) exists a unique solution $x(t, \xi)$ on $t>0$ for any given initial data $\{x(\theta):-\tau \leq \theta \leq 0\}=\xi \in C_{\mathcal{F}_{0}}^{b}\left([-\tau, 0] ; R^{n}\right)$, moreover, both $f(x, y, t)$ and $\sigma(x, y, t)$ are locally bounded in $(x, y)$ and uniformly bounded in t. If there are functions $V \in C^{2,1}\left(R^{n} \times\right.$ $\left.R^{+} ; R^{+}\right), \beta \in L^{1}\left(R^{+} ; R^{+}\right)$and $\omega_{1}, \omega_{2} \in C\left(R^{n} ; R^{+}\right)$such that

(i) $\quad \mathcal{L} V(x, y, t) \leq \beta(t)-\omega_{1}(x)+\omega_{2}(y), \quad(x, y, t) \in R^{n} \times R^{n} \times R^{+}$,

(ii) $\omega_{1}(x)>\omega_{2}(x), \quad \forall x \neq 0$,

(iii) $\lim _{\|x\| \rightarrow \infty} \inf _{0 \leq t \leq \infty} V(x, t)=\infty$,

then

$$
\lim _{x \rightarrow \infty} x(t ; \xi)=0 \quad \text { a.s. }
$$

for every $\xi \in C_{\mathcal{F}_{0}}^{b}\left([-\tau, 0] ; R^{n}\right)$.

This lemma is called the LaShall-type invariance principle. 
Lemma 2 [23] Let $x, y \in R^{n}$. Then

$$
2 x^{T} y \leq \epsilon x^{T} x+\epsilon^{-1} y^{T} y
$$

for any $\epsilon>0$.

\section{Main result}

In order to realize synchronization of the neutral-type coupled neural networks by adaptive pinning control, some controllers are added to some of the nodes. Without loss of generality, let the first $l$ nodes be controlled, and the controllers are chosen as follows:

$$
u_{i}(t)= \begin{cases}-k_{i}(t)\left(e_{i}(t)-D e_{i}(t-\tau(t))\right), & i=1,2, \ldots, l, \\ 0, & i=l+1, l+2, \ldots, N .\end{cases}
$$

Then the error system (4) can be described by

$$
\left\{\begin{array}{l}
d\left[e_{i}(t)-D e_{i}(t-\tau(t))\right]=\left\{-C e_{i}(t)+A f\left(e_{i}(t)\right)+B g\left(e_{i}(t-\tau(t))\right)+\sum_{j=1}^{N} g_{i j} \Gamma e_{j}(t)\right. \\
\left.\quad-k_{i}(t)\left(e_{i}(t)-D e_{i}(t-\tau(t))\right)\right\} d t+\sigma\left(t, e_{i}(t), e_{i}(t-\tau(t))\right) d B(t), \\
\quad i=1,2, \ldots, l, \\
d\left[e_{i}(t)-D e_{i}(t-\tau(t))\right]=\left\{-C e_{i}(t)+A f\left(e_{i}(t)\right)+B g\left(e_{i}(t-\tau(t))\right)\right. \\
\left.\quad+\sum_{j=1}^{N} g_{i j} \Gamma e_{j}(t)\right\} d t+\sigma\left(t, e_{i}(t), e_{i}(t-\tau(t))\right) d B(t), \\
i=l+1, l+2, \ldots, N .
\end{array}\right.
$$

Our object is to design an adaptive controller such that the neutral-type coupled neural network (2) can realize synchronization. The main results are stated as follows.

Theorem 1 Suppose that Assumptions 1-3 hold. The controlled network (2) can be synchronized with the trajectory of $s(t)$ for almost every initial data, if there exist positive constants $\epsilon_{i}(i=1,2,3,4), \rho, \xi_{1}, \xi_{2}$, and positive definite matrices $P, Q$, such that

(i) $\quad P \leq \rho I_{n}$,

(ii) $\Omega=\left[\begin{array}{cc}\Sigma_{1} & \Sigma_{2} \\ \Sigma_{2}^{T} & \Sigma_{3}\end{array}\right] \leq 0$

(iii) $\xi_{1}>\xi_{2}$,

where

$$
\begin{aligned}
\Sigma_{1}= & I_{N} \otimes\left(Q+\frac{1}{2}\left[-P C-C^{T} P+\epsilon_{1} P A^{T} A P+\epsilon_{2} P B B^{T} P\right.\right. \\
& \left.\left.+\left(\epsilon_{1}^{-1} l_{1}^{2}+\epsilon_{3} l_{1}^{2}+\rho h_{1}+2 \xi_{1}\right) I_{n}\right]\right)+c G \otimes P \Gamma-K^{*} \otimes P, \\
\Sigma_{2}= & \frac{1}{2} I_{N} \otimes D^{T} P C+K^{*} \otimes D^{T} P-\frac{c}{2} G \otimes D^{T} P \Gamma, \\
\Sigma_{3}= & I_{N} \otimes\left(-(1-\bar{\tau}) Q+\frac{1}{2}\left[\epsilon_{3}^{-1} D^{T} P A A^{T} P D+\epsilon_{4}^{-1} D^{T} P B B^{T} P D\right.\right.
\end{aligned}
$$




$$
\begin{aligned}
& \left.\left.+\left(\epsilon_{2}^{-1} l_{2}^{2}+\epsilon_{4}^{-1} l_{2}^{2}+\rho h_{2}-2 \xi_{2}\right) I_{n}\right]\right)-K^{*} \otimes D^{T} P D, \\
K^{*}= & \operatorname{diag}\left\{k_{1}^{*}, k_{2}^{*}, \ldots, k_{l}^{*}, 0, \ldots, 0\right\},
\end{aligned}
$$

and we choose the adaptive law as

$$
\dot{k}_{i}(t)=\alpha_{i}\left(e_{i}(t)-D e_{i}(t-\tau(t))^{T}\right) P\left(e_{i}(t)-D e_{i}(t-\tau(t))\right), \quad \alpha_{i}>0, i=1,2, \ldots, l .
$$

Proof Construct the following Lyapunov function:

$$
V(t, X)=\frac{1}{2} \sum_{i=1}^{N} X_{i}^{T} P X_{i}+\sum_{i=1}^{N} \int_{t-\tau(t)}^{t} e_{i}^{T}(s) Q e_{i}(s) d s+\sum_{i=1}^{l} \frac{1}{2 \alpha_{i}}\left(k_{i}(t)-k_{i}^{*}\right)^{2},
$$

where $X_{i}=e_{i}(t)-D e_{i}(t-\tau(t))$.

Computing $\mathcal{L} V(t, X)$ along the trajectory of error system (6), one obtains

$$
\begin{aligned}
\mathcal{L} V(t, X)= & \sum_{i=1}^{N}\left[e_{i}^{T}(t) Q e_{i}(t)-(1-\dot{\tau}(t)) e_{i}^{T}(t-\tau(t)) Q e_{i}(t-\tau(t))\right] \\
& +\sum_{i=1}^{l} \frac{1}{\alpha_{i}}\left(k_{i}(t)-k_{i}^{*}\right) \dot{k}_{i}(t) \\
& +\sum_{i=1}^{N} X_{i}^{T} P\left[-C e_{i}(t)+A f\left(e_{i}(t)\right)+B g\left(e_{i}(t-\tau(t))\right)+\sum_{j=1}^{N} g_{i j} \Gamma e_{j}(t)\right] \\
& -\sum_{i=1}^{l} k_{i}(t) X_{i}^{T} P\left(e_{i}(t)-D e_{i}(t-\tau(t))\right) \\
& +\sum_{i=1}^{N} \frac{1}{2} \operatorname{trace}\left(\sigma^{T}\left(t, e_{i}(t), e_{i}(t-\tau(t))\right) \operatorname{P\sigma }\left(t, e_{i}(t), e_{i}(t-\tau(t))\right)\right) .
\end{aligned}
$$

Based on Lemma 2 and Assumption 1, we have

$$
\begin{aligned}
e_{i}^{T}(t) P A f\left(e_{i}(t)\right) & \leq \frac{1}{2} \epsilon_{1} e_{i}^{T}(t) P A A^{T} P e_{i}(t)+\frac{1}{2} \epsilon_{1}^{-1} f^{T}\left(e_{i}(t)\right) f\left(e_{i}(t)\right) \\
& \leq \frac{1}{2} e_{i}^{T}(t)\left(\epsilon_{1} P A A^{T} P+\epsilon_{1}^{-1} l_{1}^{2} I_{n}\right) e_{i}(t) .
\end{aligned}
$$

By following similar steps to those denoted in (9), we get

$$
\begin{aligned}
& e_{i}^{T}(t) P B g\left(e_{i}(t-\tau(t))\right) \leq \frac{1}{2} \epsilon_{2} e_{i}^{T}(t) P B B^{T} P e_{i}(t)+\frac{1}{2} \epsilon_{2}^{-1} l_{2}^{2} e_{i}^{T}(t-\tau(t)) e_{i}(t-\tau(t)) \\
& -e_{i}^{T}(t-\tau(t)) D^{T} P A f\left(e_{i}(t)\right) \\
& \quad \leq \frac{1}{2} \epsilon_{3} l_{1}^{2} e_{i}^{T}(t) e_{i}(t)+\frac{1}{2} \epsilon_{3}^{-1} e_{i}^{T}(t-\tau(t)) D^{T} P A A^{T} P D e_{i}(t-\tau(t)) \\
& -e_{i}^{T}(t-\tau(t)) D^{T} P B g\left(e_{i}(t-\tau(t))\right) \\
& \quad \leq \frac{1}{2} e_{i}^{T}(t-\tau(t))\left(\epsilon_{4} D^{T} P B B^{T} P D+\epsilon_{4}^{-1} l_{2}^{2} I_{n}\right) e_{i}(t-\tau(t)) .
\end{aligned}
$$


According to Assumption 2 and $P<\rho I_{n}$, we obtain

$$
\begin{aligned}
& \sum_{i=1}^{N} \frac{1}{2} \operatorname{trace}\left(\sigma^{T}\left(t, e_{i}(t), e(t-\tau(t))\right) P \sigma\left(t, e_{i}(t), e(t-\tau(t))\right)\right) \\
& \leq \sum_{i=1}^{N} \frac{1}{2} \rho\left[h_{1} e_{i}^{T}(t) e_{i}(t)+h_{2} e_{i}^{T}(t-\tau(t)) e_{i}(t-\tau(t))\right]
\end{aligned}
$$

Substituting (7) one has the following expression:

$$
\begin{aligned}
& \sum_{i=1}^{l} \frac{1}{\alpha_{i}}\left(k_{i}(t)-k_{i}^{*}\right) \dot{k}_{i}(t)-\sum_{i=1}^{l} k_{i}(t) X_{i}^{T} P\left(e_{i}(t)-D e_{i}(t-\tau(t))\right) \\
& =-\sum_{i=1}^{l} k_{i}^{*}\left(e_{i}(t)-D e_{i}(t-\tau(t))\right)^{T} P\left(e_{i}(t)-D e_{i}(t-\tau(t))\right) \\
& =-\sum_{i=1}^{l} k_{i}^{*}\left[e_{i}^{T}(t) P e_{i}(t)-2 e_{i}^{T}(t-\tau(t)) D^{T} P e_{i}(t)\right. \\
& \left.\quad+e_{i}^{T}(t-\tau(t)) D^{T} P D e_{i}(t-\tau(t))\right]
\end{aligned}
$$

Substituting (9)-(14) into (8), one can verify easily that

$$
\begin{aligned}
\mathcal{L} V( & , X) \\
\leq & \sum_{i=1}^{N} e_{i}^{T}(t)\left\{\left(Q+\frac{1}{2}\left[-P C-C^{T} P+\epsilon_{1} P A^{T} A P+\epsilon_{2} P B B^{T} P\right.\right.\right. \\
& \left.\left.\left.+\left(\epsilon_{1}^{-1} l_{1}^{2}+\epsilon_{3} l_{1}^{2}+\rho h_{1}\right) I_{n}\right]\right) e_{i}(t)+c \sum_{j=1}^{N} g_{i j} P \Gamma e_{j}(t)\right\}-\sum_{i=1}^{l} k_{i}^{*} e_{i}^{T}(t) P e_{i}(t) \\
& +\sum_{i=1}^{N} e_{i}^{T}(t-\tau(t))\left\{\left(D^{T} P C+2 k_{i}^{*} D^{T} P\right) e_{i}(t)-c \sum_{j=1}^{N} g_{i j} D^{T} P \Gamma e_{j}(t)\right\} \\
& +\sum_{j=1}^{N} e_{i}^{T}(t-\tau(t))\left\{-(1-\bar{\tau}) Q+\frac{1}{2}\left[\epsilon_{3}^{-1} D^{T} P A A^{T} P D+\epsilon_{4}^{-1} D^{T} P B B^{T} P D\right.\right. \\
& \left.\left.+\left(\epsilon_{2}^{-1} l_{2}^{2}+\epsilon_{4}^{-1} l_{2}^{2}+\rho h_{2}\right) I_{n}\right]\right\} e_{i}(t-\tau(t))-\sum_{i=1}^{l} k_{i}^{*} e_{i}^{T}(t-\tau(t)) D^{T} P D e_{i}(t-\tau(t)) \\
& \left.\left.\left.+\left(\epsilon_{2}^{-1} l_{2}^{2}+\epsilon_{4}^{-1} l_{2}^{2}+\rho h_{2}-2 \xi_{2}\right) I_{n}\right]\right)-K^{*} \otimes D^{T} P D\right) e(t-\tau(t)) \\
& +e^{T}(t-\tau(t))\left(I _ { N } \otimes \left(-(1-\bar{\tau}) Q+\frac{1}{2}\left[\epsilon_{3}^{-1} D^{T} P A A^{T} P D+\epsilon_{4}^{-1} D^{T} P B B^{T} P D\right.\right.\right. \\
& +e^{T}(t)\left\{I _ { N } \otimes \left(Q+\frac{1}{2}\left[-P C-C^{T} P+\epsilon_{1} P A^{T} A P+\epsilon_{2} P B B^{T} P\right.\right.\right. \\
& \left.\left.\left.+\left(\epsilon_{1}^{-1} l_{1}^{2}+\epsilon_{3} l_{1}^{2}+\rho h_{1}+2 \xi_{1}\right) I_{n}\right]\right)+c G \otimes P \Gamma-K^{*} \otimes P\right\} e(t) \\
& \left.+I_{N} \otimes D^{T} P C+2 K^{*} \otimes D^{T} P-c G \otimes D^{T} P \Gamma\right) e(t)
\end{aligned}
$$




$$
\begin{aligned}
& -\xi_{1} e^{T}(t) e(t)+\xi_{2} e^{T}(t-\tau(t)) e(t-\tau(t)) \\
= & \zeta^{T}(t) \Omega \zeta(t)-\xi_{1} e^{T}(t) e(t)+\xi_{2} e^{T}(t-\tau(t)) e(t-\tau(t)),
\end{aligned}
$$

where $\zeta(t)=\left[e^{T}(t), e^{T}(t-\tau(t))\right]^{T}, e(t)=\left[e_{1}^{T}(t), e_{2}^{T}(t), \ldots, e_{N}^{T}(t)\right]^{T}$, and $K^{*}=\operatorname{diag}\left\{k_{1}^{*}, \ldots, k_{l}^{*}\right.$, $0, \ldots, 0\}$.

Considering $\Omega<0$, we have

$$
\mathcal{L} V(t, X) \leq-\xi_{1} e^{T}(t) e(t)+\xi_{2} e^{T}(t-\tau(t)) e(t-\tau(t))=-\omega_{1}(e(t))+\omega_{2}(e(t-\tau(t))),
$$

where $\omega_{1}(e(t))=\xi_{1} e^{T}(t) e(t)$ and $\omega_{2}(e(t-\tau(t)))=\xi_{2} e^{T}(t-\tau(t)) e(t-\tau(t))$.

It can be seen that $\omega_{1}(e(t))>\omega_{2}(e(t))$ for any $e(t) \neq 0$. Therefore, applying a LaSalle-type invariance principle for the stochastic differential delay equation, we can conclude that the controlled network (2) can be synchronized with the trajectory $s(t)$ for almost every initial data. The proof is completed.

Remark 1 In this paper, we investigate the almost surely synchronization for neutral-type coupled neural networks by adding adaptive controllers to the partial nodes. It is different from that in [27], where the authors consider the exponential synchronization in mean square of the coupled complex dynamical networks by adding adaptive controllers to all nodes.

When $D=0$, from Theorem 1 we obtain the following corollary.

Corollary 1 Suppose that Assumptions 1-3 hold. The controlled network (2) with D =0 can be synchronized with the trajectory of $s(t)$ for almost every initial data, if there exist positive constants $\epsilon_{i}(i=1,2), \rho, \xi_{1}, \xi_{2}$, and positive definite matrices $P, Q$, such that

(i) $\quad P \leq \rho I_{n}$

(ii) $I_{N} \otimes\left(\frac{1}{2}\left[-P C-C^{T} P+\epsilon_{1} P A^{T} A P+\epsilon_{2} P B B^{T} P+\left(\epsilon_{1}^{-1} l_{1}^{2}+\rho h_{1}+2 \xi_{1}\right) I_{n}\right]\right)$

$+c G \otimes P \Gamma-K^{*} \otimes P \leq 0$,

(iii) $\xi_{1}>\xi_{2}$,

where $\xi_{2}=\frac{1}{2}\left(\epsilon_{2}^{-1} l_{2}^{2}+\rho h_{2}\right)$ and $K^{*}=\operatorname{diag}\left\{k_{1}^{*}, k_{2}^{*}, \ldots, k_{l}^{*}, 0, \ldots, 0\right\}$. We choose the adaptive law as

$$
\dot{k}_{i}(t)=\alpha_{i} e_{i}^{T}(t) P e_{i}(t), \quad \alpha_{i}>0, i=1,2, \ldots, l .
$$

When the time-varying delay is constant $($ i.e. $\tau(t)=\tau)$, from Theorem 1 we have the following corollary.

Corollary 2 Suppose that Assumptions 1-3 hold. The controlled network (2) can be synchronized with the trajectory of $s(t)$ for almost every initial data, if there exist positive con- 


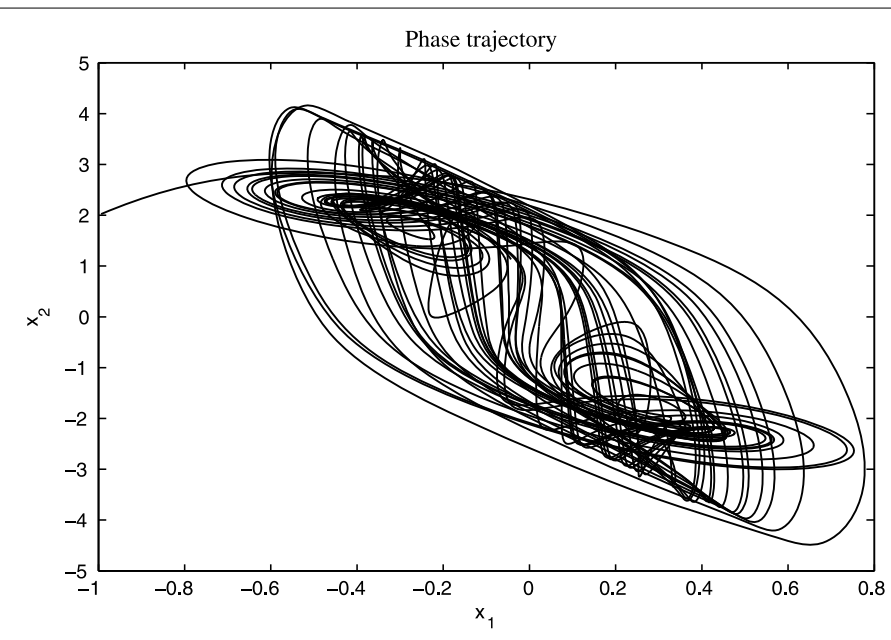

Figure 1 Dynamical behavior of the neutral-type system (3).

stants $\epsilon_{i}(i=1,2,3,4), \rho, \xi_{1}, \xi_{2}$, and positive definite matrices $P, Q$, such that

(i) $\quad P \leq \rho I_{n}$,

(ii) $\Omega=\left[\begin{array}{cc}\Sigma_{1} & \Sigma_{2} \\ \Sigma_{2}^{T} & \Sigma_{3}\end{array}\right] \leq 0$,

(iii) $\xi_{1}>\xi_{2}$,

where

$$
\begin{aligned}
\Sigma_{1}= & I_{N} \otimes\left(Q+\frac{1}{2}\left[-P C-C^{T} P+\epsilon_{1} P A^{T} A P+\epsilon_{2} P B B^{T} P\right.\right. \\
& \left.\left.+\left(\epsilon_{1}^{-1} l_{1}^{2}+\epsilon_{3} l_{1}^{2}+\rho h_{1}+2 \xi_{1}\right) I_{n}\right]\right)+c G \otimes P \Gamma-K^{*} \otimes P, \\
\Sigma_{2}= & \frac{1}{2} I_{N} \otimes D^{T} P C+K^{*} \otimes D^{T} P-\frac{c}{2} G \otimes D^{T} P \Gamma, \\
\Sigma_{3}= & I_{N} \otimes\left(-Q+\frac{1}{2}\left[\epsilon_{3}^{-1} D^{T} P A A^{T} P D+\epsilon_{4}^{-1} D^{T} P B B^{T} P D\right.\right. \\
& \left.\left.+\left(\epsilon_{2}^{-1} l_{2}^{2}+\epsilon_{4}^{-1} l_{2}^{2}+\rho h_{2}-2 \xi_{2}\right) I_{n}\right]\right)-K^{*} \otimes D^{T} P D, \\
K^{*}= & \operatorname{diag}\left\{k_{1}^{*}, k_{2}^{*}, \ldots, k_{l}^{*}, 0, \ldots, 0\right\},
\end{aligned}
$$

and we choose the adaptive law as

$$
\dot{k}_{i}(t)=\alpha_{i}\left(e_{i}(t)-D e_{i}(t-\tau)^{T}\right) P\left(e_{i}(t)-D e_{i}(t-\tau)\right), \quad \alpha_{i}>0, i=1,2, \ldots, l .
$$

\section{Numerical simulation}

In this section, we present a numerical simulation to illustrate the feasibility and effectiveness of our results. 

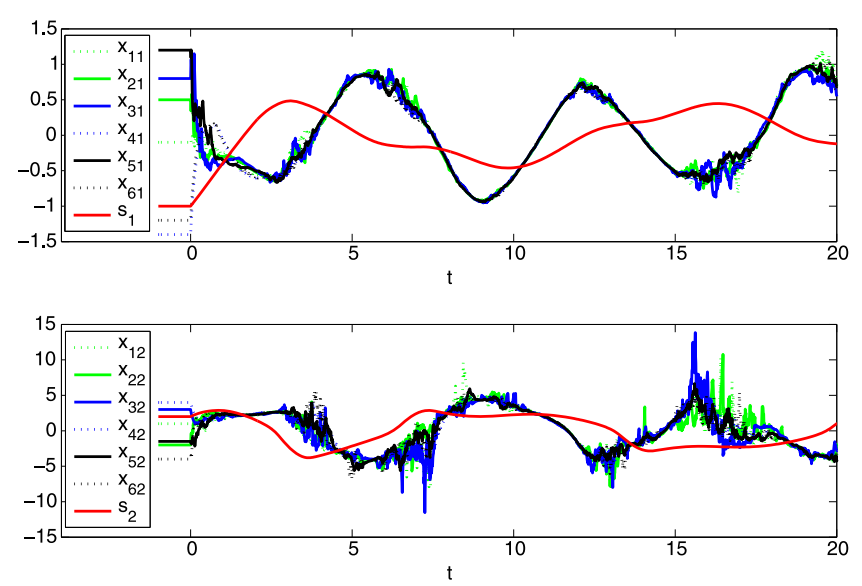

Figure 2 The trajectories of the state variables of $x_{i}(t)(i=1, \ldots, 6)$ in the uncontrolled network (2).

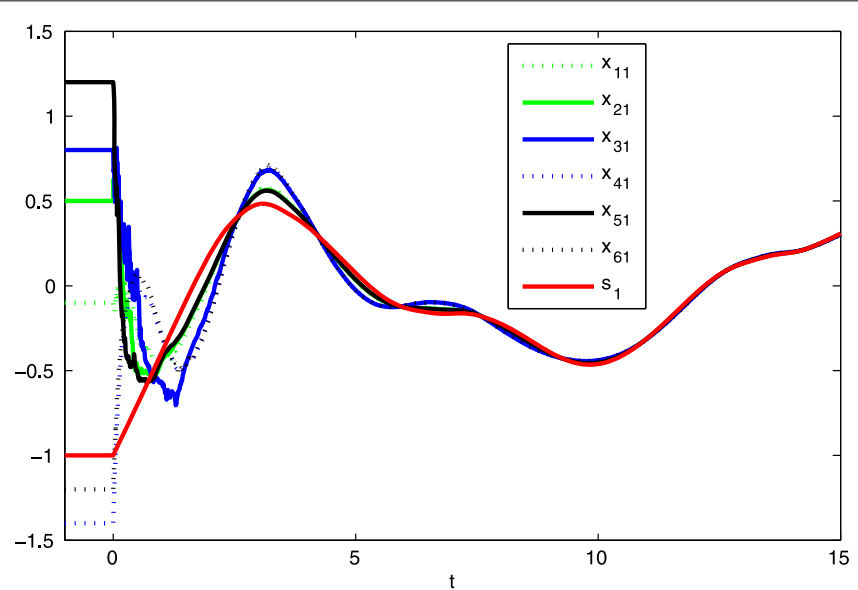

Figure 3 The trajectories of the state variables of $x_{i 1}(t)(i=1,2, \ldots, 6)$ in the controlled network (2).

Consider the neutral-type coupled neural network (2) consisting of six nodes and the parameters given as follows:

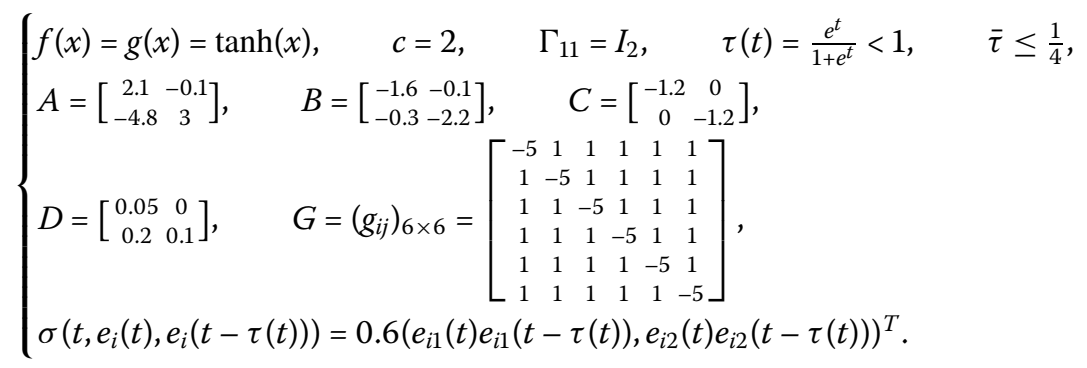

The nonlinear functions $f(\cdot)$ and $g(\cdot)$ satisfy the Lipschitz condition with $l_{1}=l_{2}=1$. The noise intensity matrix $\sigma(\cdot, \cdot, \cdot)$ satisfies the bound condition with $h_{1}=h_{2}=0.36$. In the simulation, we add the adaptive feedback controllers to the first, second, and fifth nodes. Let $P=I_{2}, \epsilon_{1}=0.17, \epsilon_{2}=0.5, \epsilon_{3}=0.7, \varepsilon_{4}=1.94, K^{*}=\operatorname{diag}(7,7,0,0,7,0), Q=\left[\begin{array}{ll}3.88 & 0.68 \\ 0.68 & 2.28\end{array}\right]$, 


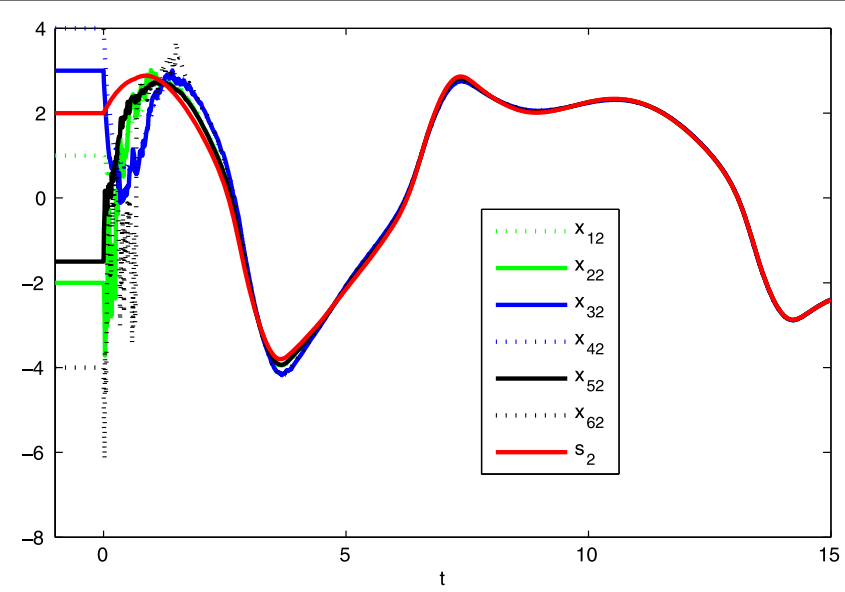

Figure 4 The trajectories of the state variables of $x_{i 2}(t)(i=1,2, \ldots, 6)$ in the controlled network (2).

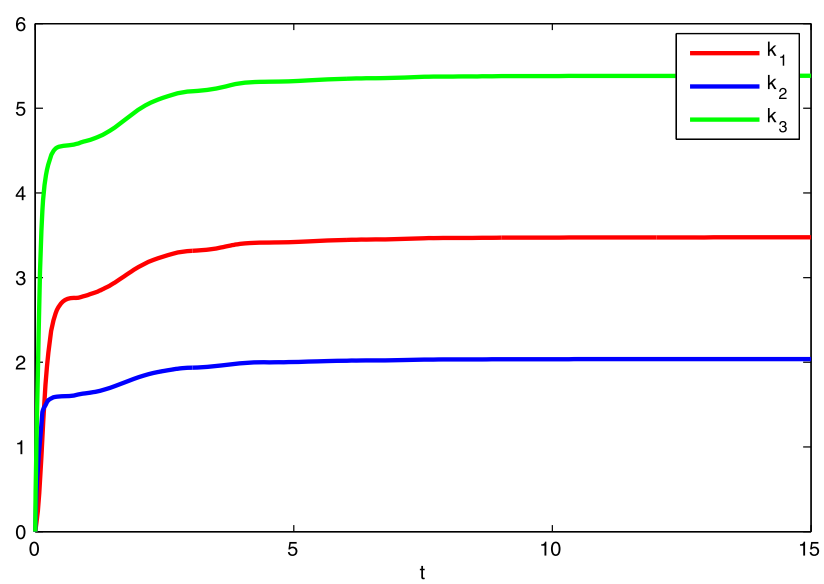

Figure 5 Time evolution of the adaptive strengths $k_{1}, k_{2}, k_{3}$.

thus

$$
\Omega<0, \quad \xi_{1}=0.2086, \quad \xi_{2}=0.1 .
$$

Hence, the condition in Theorem 1 is satisfied. According to Theorem 1, the neutral-type coupled neural network (2) achieves pinning adaptive synchronization. The initial conditions of the numerical simulation are as follows:

$$
\begin{aligned}
& X(0)=\left[x_{1}(0), x_{2}(0), \ldots, x_{6}(0)\right]=\left[\begin{array}{cccccc}
-0.1 & 0.5 & 0.8 & -1.4 & 1.2 & -1.2 \\
1 & -2 & 3 & 4 & -1.5 & -4
\end{array}\right], \\
& s(0)=\left[\begin{array}{c}
-1 \\
2
\end{array}\right], \quad k_{i}(0)=0, \quad i=1,2,3 .
\end{aligned}
$$

The simulation results are given in Figures 1-5. The dynamical behavior of the neutraltype neural network $s(t)$ is shown in Figure 1; the trajectories of the uncontrolled and controlled neutral-type coupled neural network (2) are shown in Figure 2 and Figures 3-4, 
respectively. It is clear that all nodes $x_{i}(t)$ tend to synchronization state $s(t)$ in Figures 3 and 4 . The evolution of the adaptive strengths is given as Figure 5.

\section{Conclusion}

In this paper, we have investigated the almost surely synchronization problem for an array of linearly coupled neutral-type neural networks by using adaptive pinning control. By utilizing Lyapunov stability theory and the adaptive pinning control method, some novel conditions for synchronization are derived. Furthermore, a numerical example has verified the effectiveness of the presented method.

Competing interests

The authors declare that they have no competing interests.

\section{Authors' contributions}

XS carried out the main part of this manuscript. ZF participated in the discussion and corrected the main theorem. XL read and approved the final manuscript.

\section{Author details}

${ }^{1}$ Science and Technology Information, Shaanxi Railway Institute, Weinan, 714000, P.R. China. ${ }^{2}$ Network Information Technology Center, Beifang University of Nationalities, Yinchuan, 750021, P.R. China. ${ }^{3}$ School of Electronic Engineering, Dongguan University of Technology, Dongguan, 523808, P.R. China.

\section{Acknowledgements}

This work is supported by the Scientific Research Foundation Program of Shaanxi Railway Institute (2011-27).

Received: 3 January 2014 Accepted: 25 February 2014 Published: 07 Mar 2014

\section{References}

1. Gopalsamy, K, He, X: Delay-independent stability in bidirectional associative memory networks. Automatica 23 311-326 (1987)

2. Arik, S: Stability analysis of delayed neural networks. IEEE Trans. Circuits Syst. I 47, 1089-1092 (2000)

3. Perez-Munuzuri, V, Perez-Villar, V, Chua, L: Autowaves for image processing on a two-dimensional CNN array of excitable nonlinear circuits: flat and wrinkled labyrinths. IEEE Trans. Circuits Syst. | 40, 174-181 (1993)

4. Principle, J, Kuo, J, Celebi, S: An analysis of the gamma memory in dynamic neural networks. IEEE Trans. Neural Netw. 5, 337-361 (1994)

5. Wang, Z, Shu, H, Liu, Y, Ho, D, Liu, X: Robust stability analysis of generalized neural networks with discrete and distributed time delays. Chaos Solitons Fractals 30, 886-896 (2006)

6. Wu, W, Chen, T: Global synchronization criteria of linearly coupled neural network systems with time-varying coupling. IEEE Trans. Neural Netw. 19, 319-332 (2008)

7. Lu, W, Chen, T: Synchronization of coupling connected neural networks with delays. IEEE Trans. Circuits Syst. I 51 2491-2503 (2004)

8. He, W, Cao, J: Exponential synchronization of hybrid coupled networks with delayed coupling. IEEE Trans. Neural Netw. 21, 571-583 (2010)

9. Ding, X, Gao, Y, Zhou, W, Tong, D, Su, H: Adaptive almost surely asymptotically synchronization for stochastic delayed neural networks with Markovian switching. Adv. Differ. Equ. 2013, 211 (2013)

10. Li, T, Wang, T, Yang, X, Fei, S: Pinning cluster synchronization for delayed dynamical networks via Kronecker product. Circuits Syst. Signal Process. 32, 1907-1929 (2013)

11. Wang, R, Zhang, Z, Qu, J, Cao, J: Phase synchronization motion and neural coding in dynamic transmission of neural information. IEEE Trans. Neural Netw. 22, 1097-11106 (2011)

12. Yang, $X$, Cao, J, Long, Y, Rui, W: Adaptive lag synchronization for competitive neural networks with mixed delays and uncertain hybrid perturbations. IEEE Trans. Neural Netw. 21, 1656-1667 (2010)

13. Chen, G, Zhou, J, Liu, Z: Global synchronization of coupled delayed neural networks and applications to chaotic CNN models. Int. J. Bifurc. Chaos 14, 2229-2240 (2004)

14. Li, C, Yang, S: Synchronization in linearly coupled dynamical networks with distributed time-delay coupling. Chaos 18, 2039-2047 (2008)

15. Li, Z, Fang, G, Hill, D: Controlling complex dynamical networks with coupling delays to a desired orbit. Phys. Lett. A $359,42-46(2006)$

16. Huang, T, Li, C, Liu, X: Synchronization of chaotic systems with delay using intermittent linear state feedback. Chaos $18,033122(2008)$

17. Jiang, G, Tang, W, Chen, G: A state-oberver-based approach for synchronization in complex dynamical networks. IEEE Trans. Circuits Syst. I 53, 2739-2745 (2006)

18. Dai, A, Zhou, W, Feng, J, Fang, JA, Xu, S: Exponential synchronization of the coupling delayed switching complex dynamical networks via impulsive control. Adv. Differ. Equ. 2013, 195 (2013)

19. Chen, T, Liu, X, Lu, W: Pinning complex networks by a single controller. IEEE Trans. Circuits Syst. I 54, 1317-1326 (2007)

20. Jin, $X$, Yang, G: Adaptive pinning synchronization of a class of nonlinearly coupled complex networks. Commun. Nonlinear Sci. Numer. Simul. 18, 316-326 (2013) 
21. Lee, T, Park, J, Jung, H, Lee, S, Kwon, O: Synchronization of a delayed complex dynamical network with free coupling matrix. Nonlinear Dyn. 69, 1081-1090 (2012)

22. Yang, X, Cao, J: Adaptive pinning synchronization of complex networks with stochastic perturbations. Discrete Dyn. Nat. Soc. 2010, $416182(2010)$

23. Zhu, Q, Zhou, W, Tong, D, Fang, J: Adaptive synchronization for stochastic neural networks of neutral-type with mixed time-delays. Neurocomputing 99, 477-485 (2013)

24. Lee, S, Kwon, O, Park, J: A novel delay-dependent criterion for delayed neural networks of neutral type. Phys. Lett. A 374, 1843-1848 (2010)

25. Chen, W, Wang, L: Delay-dependent stability for neutral-type neural networks with time-varying delays and Markovian jumping parameters. Neurocomputing 120, 569-576 (2013)

26. Zhou, W, Gao, Y, Tong, D, Ji, C, Fang, J: Adaptive exponential synchronization in pth moment of neutral-type neural networks with time delays and Markovian switching. Int. J. Control. Autom. Syst. 11, 845-851 (2013)

27. Zhang, Y, Gu, D, Xu, S: Global exponential adaptive synchronization of complex dynamical networks with neutral-type neural network nodes and stochastic disturbances. IEEE Trans. Circuits Syst. I 60, 2709-2718 (2013)

28. Mao, XR, Yuan, CG: Stochastic Differential Equations with Markovian Switching. Imperial College Press, London (2006)

10.1186/1687-1847-2014-77

Cite this article as: Sun et al.: Pinning adaptive synchronization of neutral-type coupled neural networks with stochastic perturbation. Advances in Difference Equations 2014, 2014:77

\section{Submit your manuscript to a SpringerOpen ${ }^{\circ}$ journal and benefit from:}

- Convenient online submission

- Rigorous peer review

- Immediate publication on acceptance

- Open access: articles freely available online

- High visibility within the field

- Retaining the copyright to your article 